\title{
Gli inibitori della pompa protonica nelle patologie acido correlate: il ruolo di rabeprazolo in una strategia di cost-minimisation
}

Giorgio L. Colombo*, Andrea Muzio*

\begin{abstract}
The clinical management of acid-related diseases incurs the consumption of a relevant part of the health resources in Italy, as indicated by the fact that PPIs (proton pump inhibitors) represented the first pharmacological class in terms of drug expenditures in 2002, making up 6,3\% of the total. It's therefore of primary importance to evaluate the economical consequences of their utilization, beyond the clinical outcomes.

In the present article a cost-minimization analysis is performed, basing on drug consumption data and focusing on the new reference-price based criteria introduced by the Italian Ministry of Health for the reimbursement of pharmaceuticals. A model for the economical evaluation of the use of PPIs was developed, based on the assumption that all the active substances in this class share the same clinical benefit, and following a stepdown approach, in which the PPIs are administered full-dose for a month, followed by a one year maintenance period at half-dose.

The model yielded the following results: rabeprazole is the most cost-saving drug of this class in the Italian setting, permitting important savings on an hypothetical 1.000 patient population, ranging from 13.000 euro, when compared to omeprazole, to 279.000 euro, if related to the most expensive substance, esomeprazole. In other terms, on the basis of the assumed equal effectiveness of the considered alternatives, the costs incurred for the treatment of 1.000 patients with esomeprazole would be sufficient to treat 1.769 patients with rabeprazole.
\end{abstract}

Farmeconomia e percorsi terapeutici 2003; 4 (1): 17-24

\section{INTRODUZIONE}

La gestione clinica di patologie acido correlate (per es. malattia da reflusso gastroesofageo, ulcera peptica e gastrite) comporta il consumo di rilevanti risorse sanitarie (visite mediche, procedure diagnostiche, ospedalizzazioni) e l'impiego di farmaci $\left(\mathrm{H}_{2} \mathrm{RA}\right.$ antagonisti- $\mathrm{H}_{2} \mathrm{RA}$; Inibitori della Pompa Protonica - IPP), le cui conseguenze devono essere attentamente valutate non solo dal punto di vista clinico, ma anche da quello economico [1]. L'esigenza di misurare il consumo dei farmaci per determinate categorie di prodotti riveste, infatti, un'importanza cruciale nel nostro sistema sanitario, al fine di definire corrette politiche di ottimizzazione della spesa sanitaria. La diffusione di sistemi informatici e di banche dati sempre più aggiornate permette infatti di sviluppare studi e ricerche di farmacoutilizzazione su vasta scala, che possono essere impiegate a supporto per interventi, ad esempio, sulla razionalizzazione della spesa farmaceutica regionale o sulle modalità prescrittive.

Nella definizione di studi di farmacoutilizzazione, la spesa, come indicatore del consumo farmaceutico, è un criterio di misurazione molto importante quando si desidera contestualizzare quest'ultimo nell' ambito del consumo sanitario in generale e in rapporto alle sue componenti (ospedaliera, personale, ecc). Si tratta, tuttavia, di un'unità di misura estremamente legata al prezzo e i cambiamenti di quest'ultimo (criteri amministrativi, costi di produzione) comportano una variazione della spesa senza che si possa parlare di un' effettiva variazione del consumo. Il numero di pezzi (confezioni) prescritti, venduti o consumati è, in altri casi, il dato di partenza normalmente a disposizione per lo sviluppo di lavori sui consumi dei farmaci.
* S.A.V.E. Studi Analisi Valutazioni Economiche, Milano 
Nella tabella 1 vengono mostrati i consumi dei farmaci in Italia nei primi 9 mesi del 2002 aggregati secondo la classificazione ATC ed espressi per spesa lorda pro-capite in DDD/ 1000 abitanti die (Defined Daily Dose). Possiamo notare come i farmaci per l'apparato gastrointestinale rappresentino, per quanto riguarda i consumi in Italia, il terzo gruppo in termini percentuali $(12,9 \%)$ per spesa lorda procapite (dopo quelli per il sistema cardiovascolare e gli antimicrobici) e il secondo gruppo per DDD per 1.000 abitanti die.

Nella tabella 2 sono riportati i primi dieci sottogruppi clinici per spesa nei primi 9 mesi degli anni 2000, 2001 e 2002. Nel periodo di tre anni preso in considerazione si nota una grossa crescita del mercato degli inibitori di pompa, che passano dal 3,8\% della spesa nel 2000 al $6,2 \%$ nel 2002, diventando il primo sottogruppo per spesa con un mercato complessivo di quasi 600 milioni di euro.

In un recente lavoro (Lucioni et al. 2002) si stima che circa l' $1 \%$ della popolazione italiana è in cura con questi farmaci, con un consumo totale in 268 milioni di DDD nel corso del 2001, il che corrisponde a circa 12 DDD per 1.000 abitanti al giorno. Questo dato appare interessante per attuare dei confronti sia internazionali sia temporali sullo stesso gruppo di farma- ci. Ad esempio il consumo degli IPP in Italia appare modesto se confrontato a quello di altri Paesi Europei; nel Regno Unito il consumo si aggira intorno alle 19,3 DDD/1.000 ab. die mentre in Francia è stimato intorno alle 16,5 DDD/ 1.000 ab. die (Lucioni et al. 2002).

\section{CONSUMO E COSTI DEGLIIPP INITALIA: ILRUOLODELLEDDD (DEFINED DAILY DOSE)}

Attualmente, trova impiego anche in Italia l'espressione del consumo di determinate categorie di farmaci in termini di Dose Definita Giornaliera (Defined Daily Dose - DDD). La DDD è la dose media di un farmaco assunta giornalmente da un paziente adulto dal peso di 70 chili, con riferimento all'indicazione terapeutica principale del farmaco stesso. Occorre sottolineare che la DDD è solo uno strumento tecnico per confrontare i consumi di farmaci, quindi, non le si deve attribuire il significato di dose consigliata o di dose effettivamente utilizzata nella pratica medica.

La DDD viene attribuita ai singoli principi attivi sulla base della classificazione ATC; in particolare all'interno di uno stesso gruppo terapeutico si avrà una DDD unica per ogni principio attivo indipendentemente dalla via

\section{Tabella 1}

Consumi farmaceutici territoriali SSN per gruppi terapeutici (ATC al I livello): confronto fra i primi 9 mesi del 2001 e del 2002

Fonte: Osservatorio Nazionale sull'Impiego di Medicinali,

Ministero della Salute, 2002

\begin{tabular}{|c|c|c|c|c|c|c|}
\hline & $\begin{array}{r}\text { Spesa lorda pro } \\
\text { capite (euro) }\end{array}$ & $\%$ & $\begin{array}{r}\% \\
02 / 01\end{array}$ & $\begin{array}{r}\text { DDD } / 1000 \\
\text { ab die }\end{array}$ & $\%$ & $\begin{array}{r}\% \\
01 / 02\end{array}$ \\
\hline C- Sistema cardiovascolare & 52,54 & 31,9 & 6 & 316,0 & 44,5 & 3 \\
\hline $\begin{array}{c}\mathrm{J} \text { - Antimicrobici generali } \\
\text { per uso sistemico }\end{array}$ & 21,91 & 13,3 & -5 & 23,9 & 3,4 & 0 \\
\hline $\begin{array}{l}\text { A - Apparato } \\
\text { gastrointestinale e } \\
\text { metabolismo }\end{array}$ & 21,26 & 12,9 & 7 & 79,2 & 11,2 & 4 \\
\hline $\begin{array}{l}\mathrm{N} \text { - Sistema nervoso } \\
\text { centrale }\end{array}$ & 14,59 & 8,9 & 25 & 36,4 & 5,1 & $\begin{array}{l}14 \\
-6\end{array}$ \\
\hline $\mathrm{R}$ - Sistema respiratorio & 12,64 & 7,7 & 2 & 54,7 & 7,7 & \\
\hline $\begin{array}{c}\text { L - Farmaci antineoplastici } \\
\text { e immunomodulatori }\end{array}$ & 9,90 & 6,0 & 7 & 5,9 & 0,8 & 6 \\
\hline $\begin{array}{c}\text { B - Sangue e organi } \\
\text { emopoietici }\end{array}$ & 9,31 & 5,7 & -1 & 57,7 & 8,1 & 11 \\
\hline $\begin{array}{l}\mathrm{H} \text { - Sistema muscolo- } \\
\text { scheletrico }\end{array}$ & 7,30 & 4,4 & -8 & 40,0 & 5,6 & 0 \\
\hline $\begin{array}{c}\text { G - Sistema genito-urinario } \\
\text { e ormoni sessuali }\end{array}$ & 7,29 & 4,4 & 2 & 40,8 & 5,8 & -3 \\
\hline $\begin{array}{l}\text { H - Preparati ormonali } \\
\text { sistemici, esclusi gli } \\
\text { ormoni sessuali }\end{array}$ & 3,27 & 2,0 & 3 & 23,2 & 3,3 & 5 \\
\hline S - Organi di senso & 2,35 & 1,4 & 12 & 19,5 & 2,7 & -1 \\
\hline D - Dermatologici & 1,43 & 0,9 & -9 & 11,8 & 1,7 & -10 \\
\hline V- Vari & 0,39 & 0,2 & 12 & 0,2 & 0,0 & 31 \\
\hline \multirow[t]{2}{*}{ P - Antiparassitari } & 0,16 & 0,1 & 1 & 0,5 & 0,1 & 0 \\
\hline & 164,48 & 100 & 4 & 709,8 & 100 & 3 \\
\hline
\end{tabular}


di somministrazione dello stesso. La DDD viene ordinariamente espressa in peso del principio attivo (g, mg, ecc.). Per esempio il rabeprazolo (ATC-A02BC) ha una DDD di 20 $\mathrm{mg}$, mentre il pantoprazolo ha una DDD di 40 $\mathrm{mg}$. La prima fonte metodologica ufficiale delle DDD è stato il Nordic Council on Medicines, la cui prima lista è stata pubblicata nel 1975 . Punto di riferimento internazionale per la metodologia delle DDD è il WHO Collaborative Centre for Drug Statistics and Methodology (WHO, 2000), mentre per il mercato italiano la fonte dei dati è rappresentata dal DURG- Italia (Drug Utilisation Research Group, 1999).

Nel quadro della distribuzione della spesa farmaceutica sopra descritta va inserita la revisione del Prontuario farmaceutico nazionale, che ha portato nuovi criteri per la determinazione del prezzo di rimborso dei farmaci [2], proprio sulla base del costo medio per DDD delle varie sostanze appartenenti a ciascuna categoria terapeutica. Tale revisione è stata condotta con l'obiettivo dichiarato di:

1) assicurare alla popolazione la copertura completa di tutte le patologie clinicamente ed epidemiologicamente rilevanti;

2) garantire ai medici di medicina generale un'adeguata possibilità di scelta tra diversi principi attivi con le stesse indicazioni terapeutiche;
3) individuare un valore di rimborso di riferimento operando un risparmio principalmente sulla marcata variabilità di prezzo registrata tra molecole di efficacia comparabile.

Di seguito si espone la metodologia adottata per il calcolo del livello di rimborso (cut-off) nell' ambito di ciascuna categoria terapeutica:

1) Nell'ambito di ciascuna categoria terapeutica è stato calcolato il costo medio giornaliero di ciascun principio attivo, ponderato sulla base dei consumi avvenuti nell'anno 2001, ovvero:

$\frac{[\operatorname{Pr} \mathrm{SSN}] \times[\text { n. Pz }]}{[\text { n. Cons }]}$

Dove:

Pr SSN: prezzo SSN 2002 vigente (CIPE)

n. Pz: numero pezzi 2001 (OsMed)

n. Cons: numero dosi consumate 2001 (OsMed)

2) Il costo medio giornaliero ponderato dei singoli principi attivi nell' ambito di ciascuna categoria è stato calcolato sulla base della Dose Definita Die (certificata OMS) e sono stati ordinati in maniera crescente;

3) Il livello di rimborso (cut-off), nell' ambito di ciascuna categoria terapeutica, è stato

\begin{tabular}{|c|c|c|c|c|c|c|c|c|}
\hline \multirow[t]{2}{*}{ Sottogruppo } & \multicolumn{2}{|c|}{ Spesa 2000} & \multirow[t]{2}{*}{ Sottogruppo } & \multicolumn{2}{|c|}{ Spesa 2001} & \multirow[t]{2}{*}{ Sottogruppo } & \multicolumn{2}{|c|}{ Spesa 2002} \\
\hline & $\begin{array}{l}\text { Euro } \\
\text { (Mil) }\end{array}$ & $\%$ & & $\begin{array}{l}\text { Euro } \\
\text { (Mil) }\end{array}$ & $\%$ & & $\begin{array}{l}\text { Euro } \\
\text { (Mil) }\end{array}$ & $\%$ \\
\hline Ace-inibitori & 443 & 6,0 & Ace-inibitori & 486 & 5,3 & Inibitori di pompa & 588 & 6,2 \\
\hline $\begin{array}{l}\text { Calcioantagonisti } \\
\text { (didropiridinici) }\end{array}$ & 442 & 6,0 & $\begin{array}{l}\text { Calcioantagonisti } \\
\text { (didropiridinici) }\end{array}$ & 483 & 5,3 & Ace-inibitori & 505 & 5,3 \\
\hline Cefalosporine & 428 & 5,8 & Inibitori di pompa & 443 & 4,9 & Statine & 483 & 5,1 \\
\hline $\begin{array}{l}\text { Ace-inibitori e diuretici } \\
\text { (associazioni) }\end{array}$ & 337 & 4,6 & Statine & 413 & 4,5 & $\begin{array}{l}\text { Calcioantagonisti } \\
\text { (didropiridinici) }\end{array}$ & 471 & 5,0 \\
\hline Inibitori di pompa & 279 & 3,8 & Cefalosporine & 401 & 4,4 & Cefalosporine & 350 & 3,7 \\
\hline Statine & 263 & 3,6 & $\begin{array}{l}\text { Ace-inibitori e diuretici } \\
\text { (associazioni) }\end{array}$ & 353 & 3,9 & $\begin{array}{l}\text { Ace-inibitori e diuretici } \\
\text { (associazioni) }\end{array}$ & 347 & 3,7 \\
\hline Macrolidi e licosamidi & 241 & 3,3 & Macrolidi e licosamidi & 267 & 2,9 & Antidepressivi SSRI & 279 & 2,9 \\
\hline Fans & 212 & 2,9 & $\begin{array}{l}\text { Antiifiammatori - } \\
\text { Cortisonici inalatori }\end{array}$ & 239 & 2,6 & Macrolidi e licosamidi & 262 & 2,8 \\
\hline $\begin{array}{l}\text { Antiifiammatori - } \\
\text { Cortisonici inalatori }\end{array}$ & 200 & 2,7 & Fans & 230 & 2,5 & $\begin{array}{l}\text { Antagonisti } \\
\text { dell'angiotensina II }\end{array}$ & 255 & 2,7 \\
\hline Nitrati & 178 & 2,4 & $\begin{array}{l}\text { Antagonisti } \\
\text { dell'angiotensina II }\end{array}$ & 225 & 2,5 & $\begin{array}{l}\text { Antiifiammatori - } \\
\text { Cortisonici inalatori }\end{array}$ & 225 & 2,4 \\
\hline
\end{tabular}

\section{Tabella 2}

Primi 10 sottogruppi clinici a maggior spesa nei primi 9 mesi degli anni 2000, 2001 e 2002

Fonte: Osservatorio Nazionale sull'Impiego di Medicinali, Ministero della Salute, 2002 
Tabella 3

Confezioni, costie DDD degli IPP in

Italia

Fonte: Informatore

Farmaceutico

Nazionale, 2003

\begin{tabular}{|c|c|c|c|c|c|c|}
\hline & $\begin{array}{r}\text { DDD } \\
\text { (mg) }\end{array}$ & $\begin{array}{l}\text { Tot mg } \\
\text { in conf }\end{array}$ & $\begin{array}{r}\text { N DDD in } \\
\text { conf }\end{array}$ & $\begin{array}{r}\text { Costo conf } \\
\text { (euro) }\end{array}$ & $\begin{array}{r}\text { Costo DDD in } \\
\text { conf (euro) }\end{array}$ & $\begin{array}{r}\text { Costo/die } \\
\text { (euro) }\end{array}$ \\
\hline \multicolumn{7}{|l|}{ Rabeprazolo } \\
\hline $10 \mathrm{mg}$ & & 140 & 7 & 12,34 & & 0,88 \\
\hline $20 \mathrm{mg}$ & 20 & 280 & 14 & 22,67 & 1,62 & 1,62 \\
\hline \multicolumn{7}{|c|}{ Pantoprazolo } \\
\hline $20 \mathrm{mg}$ & & 280 & 7 & 13,24 & & 0,95 \\
\hline $40 \mathrm{mg}$ & 40 & 560 & 14 & 26,27 & 1,88 & 1,88 \\
\hline \multicolumn{7}{|c|}{ Lansoprazolo } \\
\hline $15 \mathrm{mg}$ & & 210 & 7 & 13,90 & & 0,99 \\
\hline $30 \mathrm{mg}$ & 30 & 420 & 14 & 25,04 & 1,79 & 1,79 \\
\hline \multicolumn{7}{|l|}{ Omeprazolo } \\
\hline $10 \mathrm{mg}$ & & 140 & 7 & 12,55 & & 0,90 \\
\hline $20 \mathrm{mg}$ & 20 & 280 & 14 & 26,54 & 1,90 & 1,90 \\
\hline \multicolumn{7}{|c|}{ Esomeprazolo } \\
\hline $20 \mathrm{mg}$ & 20 & 280 & 14 & 22,67 & 1,62 & 1,62 \\
\hline $40 \mathrm{mg}$ & & 560 & 28 & 29,40 & & 2,10 \\
\hline
\end{tabular}

individuato al verificarsi contemporaneamente delle seguenti due condizioni:

a. il numero di dosi dispensate è pari ad almeno il $60 \%$ del totale cumulativo;

b. la spesa cumulata a carico del SSN è pari ad almeno il 50\% del mercato;

4) Nel caso in cui un principio attivo costituisca da solo oltre il $50 \%$ della spesa della singola categoria terapeutica (effetto dominante), non si applicano i criteri di cui al precedente punto 3 , ma il valore di rimborso è definito incrementando del $15 \%$ il prezzo medio ponderato del principio attivo che garantisce almeno il $5 \%$ cumulato del volume di mercato.

\begin{tabular}{lccr}
\hline & \% spesa & \% DDD & $\begin{array}{r}\text { Costo medio } \\
\text { DDD }\end{array}$ \\
\hline $\begin{array}{l}\text { Rabeprazolo } \\
\text { (DDD = pieno dosaggio) }\end{array}$ & 7,8 & 9,9 & 1,68 \\
$\begin{array}{l}\text { Pantoprazolo } \\
\text { (DDD = pieno dosaggio) }\end{array}$ & 11,6 & 12,9 & 1,91 \\
$\begin{array}{l}\text { Lansoprazolo } \\
\text { (DDD = pieno dosaggio) }\end{array}$ & 14,6 & 14,3 & 2,18 \\
$\begin{array}{l}\text { Omeprazolo } \\
\text { (DDD = pieno dosaggio) }\end{array}$ & 65,9 & 62,8 & 2,24 \\
\hline
\end{tabular}

\section{Tabella 4}

Costo medio DDD: dati del Ministero sulla base del mercato al 2001 Fonte: Ministero della Salute, 2002
Nella tabella 3 sono stati raccolti tutti i dati del mercato sui diversi IPP in Italia. Dall' analisi di questa tabella emergono importanti considerazioni; innanzitutto, in quattro dei cinque diversi principi attivi commercializzati, la DDD è stata fissata al quantitativo rispondente al pieno dosaggio, mentre solo per l'ultimo entrato nel mercato, l'esomeprazolo, si è fissata una DDD corrispondente al mezzo dosaggio. Il Costo DDD in confezione riportato nella tabella 3 non va confuso col costo medio per $\mathrm{DDD}$, ma è un valore costante calcolato in base al costo della confezione e al numero di DDD presenti nella stessa.

Il problema sopracitato della diversità dei criteri adottati tra i diversi IPP per la determinazione delle DDD è evidente osservando il numero totale di DDD presenti nelle confezioni. Solo l'esomeprazolo può vantare 14 DDD in una confezione da $20 \mathrm{mg}$ e 28 in quella da 40 $\mathrm{mg}$, corrispondenti rispettivamente al mezzo e al pieno dosaggio, tutti gli altri farmaci considerati hanno rispettivamente 7 e 14 DDD a confezione. Un altro fattore che rende difficile il confronto tra diversi IPP è l'effettiva diversa "potenza" dei principi attivi: alcuni sono somministrati a $10 \mathrm{mg}$ per il mezzo dosaggio e 20 per il pieno, altri rispettivamente a 15 e $30 \mathrm{mg}$, altri ancora a 20 e $40 \mathrm{mg}$. Questo tipo di disomogeneità nei dosaggi non permette di fare confronti utilizzando il costo/mg delle diverse sostanze, in quanto non indicativo del reale utilizzo del farmaco. 
Nell'ultima colonna della tabella 3, viene calcolato invece il costo/die dei diversi IPP, ricavato dal costo di una confezione (Informatore Farmaceutico Nazionale, 2003) diviso per la durata della stessa (in questo caso corrispondente al numero di compresse, uguale a 14 per tutti i farmaci), sulla base del dosaggio indicato in scheda tecnica (per ulcera gastrica e MRGE) e dall' analisi dei principali dati di letteratura. È possibile osservare quindi che un trattamento a $20 \mathrm{mg}$ (pieno dosaggio) a base di rabeprazolo presenta un costo identico a quello dell'esomeprazolo a $20 \mathrm{mg}$, che costituisce però il mezzo dosaggio.

Nella tabella 4 vengono ripresi i dati calcolati dal Ministero della Salute sulla base del mercato al 2001, secondo la metodologia introdotta per il calcolo del cut-off. Essendo ora presente nel mercato un principio attivo che rappresenta da solo oltre il $50 \%$ della spesa (effetto dominante, in questo caso omeprazolo) il valore di rimborso è definito incrementando del $15 \%$ il prezzo medio ponderato del principio attivo che garantisce almeno il $5 \%$ cumulato del volume di mercato: al 2001 il prezzo di riferimento era quello di rabeprazolo e quindi il cutoff fissato a 1,93 euro $(1,68 \times 1,15)$, con un risparmio per il SSN stimato in oltre 64,5 milioni di euro [3]. Inoltre, una stima sui dati di mercato dei primi 9 mesi del 2002 indica una sostanziale equivalenza dei costi medi DDD delle quattro sostanze, rispetto a quanto già calcolato dal Ministero.

L'evoluzione del mercato lascia pensare che presto omeprazolo, a seguito dell'introduzione di esomeprazolo, dovrà lasciare la posizione dominante all'interno di questa categoria, determinando, secondo il decreto ministeriale, un cambio nel criterio di determinazione del cut-off, che verrà calcolato secondo il criterio della spesa.

Sino a quando il numero di dosi dispensate di omeprazolo sarà superiore al 40\%, il prezzo limite sarà il prezzo stesso di omeprazolo (al momento del calcolo ministeriale 2,24 euro), azzerando completamente il risparmio per il SSN; con ulteriori diminuzioni del volume di vendite di omeprazolo il cut-off, sempre secondo il criterio della spesa, passerà prima al prezzo di lansoprazolo, poi a quello di pantoprazolo, poi ancora a quello di rabeprazolo, in funzione delle quote acquisite dalla nuova sostanza entrata nel mercato, esomeprazolo, con risparmi per il SSN nulli o comunque più contenuti, rispetto alla situazione prevista dal Ministero.

Considerando che la maggior parte degli studi clinici condotti su esomeprazolo sono stati sviluppati su dosaggi a $40 \mathrm{mg}[4,5]$ (pieno dosaggio) è stato possibile stimare anche il

\begin{tabular}{|c|c|c|c|c|c|c|}
\hline & Costo die & $\begin{array}{r}\text { Costo terapia } \\
4 \text { settimane }\end{array}$ & $\begin{array}{r}\text { Costo terapia } \\
\text { mantenimento } \\
1 \text { anno (360gg) }\end{array}$ & $\begin{array}{r}\text { Costo terapia } \\
1000 \text { pazienti } \\
(4 \text { sett }+1 \text { anno })\end{array}$ & $\begin{array}{r}\text { Risparmio } \\
\text { Rabeprazolo vs }\end{array}$ & $\begin{array}{l}\text { Pazient } \\
\text { trattabili in più }\end{array}$ \\
\hline \multicolumn{7}{|l|}{ Rabeprazolo } \\
\hline $10 \mathrm{mg}$ & 0,88 & - & 317,31 & & & \\
\hline $20 \mathrm{mg}$ & 1,62 & 45,34 & - & 362,654 & - & . \\
\hline \multicolumn{7}{|l|}{ Pantoprazolo } \\
\hline $20 \mathrm{mg}$ & 0,95 & - & 340,46 & & & \\
\hline $40 \mathrm{mg}$ & 1,88 & 52,54 & - & 392,997 & 30,343 & 83 \\
\hline \multicolumn{7}{|l|}{ Lansoprazolo } \\
\hline $15 \mathrm{mg}$ & 0,99 & - & 357,43 & & & \\
\hline $30 \mathrm{mg}$ & 1,79 & 50,08 & - & 407,509 & 44,854 & 123 \\
\hline \multicolumn{7}{|l|}{ Omeprazolo } \\
\hline $10 \mathrm{mg}$ & 0,90 & - & 322,71 & & & \\
\hline $20 \mathrm{mg}$ & 1,90 & 53,08 & - & 375,794 & 13,140 & 36 \\
\hline \multicolumn{7}{|c|}{ Esomeprazolo } \\
\hline $20 \mathrm{mg}$ & 1,62 & - & 582,94 & & & \\
\hline $40 \mathrm{mg}$ & 2,10 & 58,80 & - & 641,743 & 279,089 & 769 \\
\hline
\end{tabular}

Tabella 5

Confronti tra IPP nelle malattie acido correlate: il ruolo di rabeprazolo in una strategia di cost-minimisation (valori in euro) 
costo medio DDD qualora questa sostanza avesse avuto una DDD omogenea rispetto alle altre appartenenti allo stesso gruppo terapeutico: i risultati mostrano che il suo costo medio DDD sarebbe di 2,50 euro.

\section{CONFRONTITRA IPP NELLEMALATTIEACIDOCORRELATE}

Appare opportuno, alla luce di quanto emerso in precedenza, sviluppare un confronto farmacoeconomico nelle malattie acido correlate, al fine di verificare l'effettivo costo della terapia a base dei diversi farmaci analizzati, sulla base di una strategia di costminimisation. In tale analisi si ipotizza una strategia che prevede l'intervento sullo stato acuto della patologia con quattro settimane di trattamento a pieno dosaggio a cui si aggiunge un anno di mantenimento a mezzo dosaggio per la prevenzione dei sintomi maggiormente avvertiti; il modello proposto viene esteso al trattamento di mille pazienti. La particolarità di queste patologie e delle relative strategie di cura è proprio l'uso dei farmaci IPP a diversi dosaggi a seconda dello stadio/ caratteristiche della malattia, per questo motivo si è inteso calcolare un costo/die sulla base dei prezzi di mercato, seguendo un approccio di tipo Step-Down [6].

I risultati ottenuti (tabella 5) evidenziano che il rabeprazolo presenta un costo die inferiore a tutti gli altri inibitori della pompa protonica, pari a 0,88 euro per il trattamento a mezzo dosaggio e di 1,62 euro per l'intervento sullo stato acuto con un dosaggio di $20 \mathrm{mg}$.

Nella costruzione del modello si è ipotizzato, come già ricordato, la medesima efficacia clinica dei cinque IPP considerati. Esistono in letteratura numerose reviews che confrontano l'efficacia clinica dei diversi IPP [7]: in particolare il lavoro di Stedman e Barclay [8] ha confrontato i risultati emersi da 14 lavori in termini di efficacia clinica di diversi IPP per il trattamento della MRGE, evidenziando come per la maggior parte degli studi sia stata dimostrata sul campo una medesima risposta al farmaco sia in acuto sia per il mantenimento. Uno studio accurato della letteratura sull' argomento mostra come sia diffusa la consapevolezza nella classe medica di una simile efficacia fra i diversi IPP $[9,10]$. In generale, dosi standard di IPP (20 mg per omeprazolo, $30 \mathrm{mg}$ per lansoprazolo, $40 \mathrm{mg}$ per pantoprazolo, $20 \mathrm{mg}$ per rabeprazolo e $40 \mathrm{mg}$ per esomeprazolo) somministrate prima di colazione alleviano i sintomi e curano l'esofagite di ogni grado in circa l' 85 - $90 \%$ dei pazienti [11].
Il modello proposto nella tabella 5 risulta applicabile anche per la cura della NERD (NonErosive Reflux Disease), il cui trattamento è autorizzato in scheda tecnica per rabeprazolo ed esomeprazolo. La NERD costituisce una patologia estremamente diffusa, rappresentando circa il 50-60\% delle MRGE; nel confronto fra le due sostanze commercializzate emerge ancora più marcato il ruolo di rabeprazolo come costsaving, nell' approccio medico alla patologia.

Sulla base dell'evidenza della letteratura clinica è quindi possibile affrontare lo studio degli IPP dal punto di vista di una strategia di cost-minimisation, in quanto si suppone la medesima efficacia clinica nell' azione sui sintomi e sulle lesioni delle patologie acido correlate e nella prevenzione di ricadute grazie all'uso degli stessi a dosaggio pieno o mezzo a seconda delle indicazioni. Il modello così costruito ha permesso di calcolare il risparmio dato dall'uso di rabeprazolo $v s$ altri IPP, in 1.000 pazienti a cui viene prescritto tanto nella fase acuta quanto nel mantenimento. Dai risultati illustrati nella tabella 5 si può facilmente osservare come rabeprazolo risulti cost-saving rispetto a tutti gli altri principi attivi confrontati; in particolare il risparmio va da oltre 13 mila euro $v s$ omeprazolo fino a 279 mila euro se confrontato all'esomeprazolo. È possibile a questo punto anche calcolare quanti pazienti si potrebbero trattare con rabeprazolo, grazie al risparmio dato dal suo minor costo. I risultati mostrano che sostituendo una terapia a base di omeprazolo con quella a base di rabeprazolo, si possono trattare fino a 36 pazienti in più e questo dato sale fino ai 769 pazienti trattabili in più rispetto all'esomeprazolo: in pratica significa che con il costo del trattamento di 1.000 pazienti con esomeprazolo è possibile trattare 1.769 pazienti con rabeprazolo.

Questi dati si rivelano ancora più interessanti alla luce di quelli ottenuti da Lucioni in un recente lavoro [12], nel quale ha analizzato il Data Base Health Search, nato da un'iniziativa della Società Italiana di Medicina Italiana (SIMG) e supportato da un gruppo di aziende farmaceutiche, individuando tutte le prescrizioni di IPP effettuate da circa 350 medici. In questa ricerca, fra l'altro, l'autore ha stimato la durata media dei trattamenti (episodi prescrittivi) con i vari prodotti disponibili sul mercato: i risultati mostrano come rabeprazolo presenti durate medie, sia a mezzo che a pieno dosaggio, inferiori a tutti gli altri prodotti (rispettivamente $24,9 \mathrm{gg}$ e $37,3 \mathrm{gg}$ ).

Nella tabella 6 si è inserita questa variabile nel modello di determinazione del costo di trattamento. Il dato sulla durata media del tratta- 
mento a mezzo e a pieno dosaggio permette di calcolare il costo trattamento che, se ponderato con la distribuzione del consumo dei due diversi dosaggi, fornisce un'attendibile stima dell'ordine di grandezza del costo medio del trattamento. I risultati ottenuti sono in linea con quelli del nostro modello: il trattamento con rabeprazolo risulta cost-saving rispetto a lansoprazolo, pantoprazolo e omeprazolo (in questa analisi esomeprazolo non è stato considerato in quanto non ancora disponibili dati sulla durata del trattamento). In particolare il costo del trattamento con rabeprazolo è stato stimato in 53,09 euro, mentre le altre sostanze hanno un costo medio di oltre il $30 \%$ superiore.

\section{CONCLUSIONI}

La gestione clinica di patologie acido correlate comporta il consumo di rilevanti risorse sanitarie e l'impiego di importanti farmaci le cui conseguenze devono essere attentamente valutate non solo dal punto di vista clinico, ma anche da quello economico. La diffusione di sistemi informatici e di banche dati sempre più aggiornate permette infatti di sviluppare studi e ricerche di farmacoutilizzazione su vasta scala, che possono essere impiegate a supporto per interventi, ad esempio, sulla razionalizzazione della spesa farmaceutica regionale o sulle modalità prescrittive.

L'espressione del consumo di determinate categorie di farmaci in termini di Dose Definita Giornaliera (Defined Daily Dose - DDD), dove la DDD è la dose media di un farmaco assunta giornalmente da un paziente adulto dal peso di 70 chili, appare un ottimo strumento tecnico per confrontare i consumi di farmaci tra diversi ambiti territoriali (nazioni, regioni, ecc.) e in diversi momenti temporali. Come già osservato, uno dei problemi dell' applicazione della DDD a studi farmacoeconomici è legato al fatto che non rappresenta né una dose raccomandata né una dose effettiva, bensì è uno standard fissato ai fini di confronti in termini relativi (ad es.: totale DDD/1.000 ab.).

Quando lo scopo è quello di confrontare l'effettiva convenienza economica di diversi trattamenti, la DDD non rappresenta più uno strumento di misura ottimale in quanto, volendo esprimere i diversi volumi unitari di consumo (dose/die) per individuare fra farmaci terapeuticamente equivalenti quello a minor costo, la DDD non esprime il parametro su cui confrontare i costi poiché, come osservato, non tiene presente le reali abitudini prescrittive della realtà indagata e spesso i dosaggi giornalieri attesi con le DDD differiscono in maniera significativa da quelli effettivi.

L'impiego della PDD (Prescribed Daily Dose) nella valutazione del consumo di IPP, pur con tutti i limiti di perdita di informazione tipici di stime basate su medie statistiche, fornisce una indicazione molto più attendibile dell'effettivo utilizzo del farmaco e quindi dei costi ad esso associati, in particolare mostra come l'effettivo costo del trattamento dipenda soprattutto da come si distribuisce il consumo fra mezzo e pieno dosaggio. La difficoltà dell'applicazione corretta di una tale metodologia

\begin{tabular}{|c|c|c|c|c|c|}
\hline & Costo die & $\%$ DDD $^{1}$ & $\begin{array}{r}\text { Durata media } \\
\text { trattamento }(\mathrm{gg})[12]\end{array}$ & $\begin{array}{r}\text { Costo medio } \\
\text { trattamento }^{2}\end{array}$ & $\begin{array}{l}\% \text { costo vs } \\
\text { rabeprazolo }\end{array}$ \\
\hline \multicolumn{6}{|l|}{ Rabeprazolo } \\
\hline $\begin{array}{l}10 \mathrm{mg} \\
20 \mathrm{mg}\end{array}$ & $\begin{array}{l}0,88 \\
1,62\end{array}$ & $\begin{array}{l}19,0 \\
81,0\end{array}$ & $\begin{array}{l}24,9 \\
37,3\end{array}$ & 53,9 & 100 \\
\hline \multicolumn{6}{|l|}{ Pantoprazolo } \\
\hline $\begin{array}{l}20 \mathrm{mg} \\
40 \mathrm{mg}\end{array}$ & $\begin{array}{l}0,95 \\
1,88\end{array}$ & $\begin{array}{l}28,9 \\
71,1\end{array}$ & $\begin{array}{l}25,6 \\
52,5\end{array}$ & 77,04 & 145 \\
\hline \multicolumn{6}{|l|}{ Lansoprazolo } \\
\hline $\begin{array}{l}15 \mathrm{mg} \\
30 \mathrm{mg}\end{array}$ & $\begin{array}{l}0,99 \\
1,79\end{array}$ & $\begin{array}{l}50,7 \\
49,3\end{array}$ & $\begin{array}{l}42,3 \\
62,7\end{array}$ & 76,56 & 144 \\
\hline \multicolumn{6}{|l|}{ Omeprazolo } \\
\hline $\begin{array}{l}10 \mathrm{mg} \\
20 \mathrm{mg}\end{array}$ & $\begin{array}{l}0,90 \\
1,90\end{array}$ & $\begin{array}{r}9,9 \\
90,1\end{array}$ & $\begin{array}{l}26,9 \\
39,7\end{array}$ & 70,19 & 132 \\
\hline
\end{tabular}

${ }^{1}$ Ripartizione del numero di dosi dispensate fra mezzo e pieno dosaggio; dati di mercato, gen-sett 2002

2 Prezzi in vigore 2003

\section{Tabella 6}

Costo medio di trattamento delle malattie acido correlate con vari IPP 
risiede nella necessità di attenti studi osservazionali realizzati sul campo per poter descrivere quali siano le effettive abitudini di cura delle patologie acido correlate. Non disponendo di tali dati si è ipotizzato un consumo giornaliero simile a quello indicato dalle schede tecniche dei prodotti, suggerito anche dalla non divisibilità delle formulazioni in commercio, che indica l'uso dei farmaci in monosomministrazioni giornaliere delle compresse presenti nelle confezioni; i costi/die così ottenuti sono stati utilizzati per ricavare il costo medio ponderato per le quantità vendute nei due diversi dosaggi presenti per tutte le sostanze.

A tal proposito, è stato anche sviluppato un modello di valutazione farmacoeconomico nelle patologie acido correlate, al fine di verificare l'effettivo costo della terapia con diversi farmaci analizzati, sulla base di una strategia di cost-minimisation. Nella costruzione del modello si è ipotizzata la medesima efficacia clinica dei cinque principi attivi rientranti negli IPP, e nel contempo il trattamento di 1.000 pazienti affetti da MRGE, su cui si interviene con una strategia di tipo Step-down, con un ciclo di terapia che prevede quattro settimane di trattamento con IPP a pieno dosaggio e una terapia di mantenimento di un anno a metà dosaggio.

Il modello così costruito ha permesso di calcolare il risparmio dato dall'uso di rabeprazolo $v s$ altri IPP, in 1.000 pazienti a cui viene prescritto tanto nella fase acuta quanto nel mantenimento, con un risparmio che va da oltre 13 mila euro $v s$ omeprazolo a circa 279 mila euro se confrontato all'esomeprazolo. Ossia, con il costo di trattamento di 1.000 pazienti con esomeprazolo è possibile trattare fino a 1.769 pazienti con rabeprazolo, sulla base delle ipotesi di medesima efficacia tra le opzioni terapeutiche proposte.

Il modello, come accennato in precedenza, è stato sviluppato secondo una metodologia di cost-minimisation, che richiede l'equivalenza terapeutica tra le strategie farmacologiche considerate [13].

I vantaggi della ricerca farmacoeconomica sono di grande rilievo per il nostro SSN, anche alla luce dei recenti decreti di riordino della spesa farmaceutica. Includere nella decisione medica il rapporto costo/conseguenze economiche significa cercare di fornire al medico ulteriori strumenti per scegliere in modo razionale le risorse, dato che la discriminazione tra impieghi alternativi e soggetti che ne godranno $i$ benefici è un vincolo al quale purtroppo non è più possibile sottrarsi. Ottimizzare l'uso delle risorse in sanità significa, infatti, moltiplicare quello che possiamo fare a beneficio di tutti i pazienti.

Questo studio è stato reso possibile da un finanziamento messo a disposizione da Janssen-Cilag SpA.

\section{BIBLIOGRAFIA}

1. Colombo GL. Terapia a lungo termine nella malattia da reflusso gastroesofageo: implicazioni farmacoeconomiche, Dis Manage Health Outcomes 7 (Suppl. 1): X-XX, 2000.

2. Ministero della Salute, Supplemento ordinario alla Gazzetta ufficiale; 23/10/2002, Serie Generale n 249.

3. Ministero della Salute, Supplemento ordinario alla Gazzetta ufficiale; 23/10/2002, Serie Generale n 249.

4. Kahrilas PJ, Falk G, Whipple J, D’Amico J, Joelsson B. Comparison of esomeprazole, a novel PPI, vs omeprazole in GERD patients wth erosive esophagitis. Gastroenterol 2000, 118, A93.

5. Richter JE, Kahrilas PJ, Hwang C, Marino V, Hamelin B. Esomeprazole is superior to omeprazole for the healing of erosive esophagitis in GERD patients. Gastroenterol 2000, 118, A20.

6. Storr M, Meining A, Allescher HD. Pharmacoeconomics issues of the treatment of gastroesophageal reflux disease. Expert Opin. Pharmacother, 2001, 2(7): 1099-1108.

7. Thomson ABR. Are the orally administered proton pump inhibitors equivalent? A comparison of lansoprazole, omeprazole, pantoprazole and rabeprazole; Current Gastroenterology Reports, 2000; 2: 482-493.

8. Stedman CAM, Barclay ML. Review article: comparison of the pharmacokinetics, acid suppression and efficacy of proton pump inhibitors; Aliment Pharmacol Ther 2000; 14: 963-978.

9. Byrne MF and Murray FE. Formulary Management of Proton Pump Inhibitors. Pharmacoeconomics 1999; 16: $225-246$.

10. Tim Wright BA, Economic evaluation or rabeprazole, omeprazole and ranitidine in the treatment of acid-related upper gastrointestinal disorders; Journal of Drug Assessment, 2000; 3: 195-203.

11. Storr M, Meining A, Allescher HD. op. cit.

12. Lucioni C, Mazzi S. Realtà prescrittiva versus standard di consumo nel campo degli inibitori della pompa protonica in Italia; Pharmacoeconomics Italian Research Articles 5 (1): 3-10, 2003.

13. Barone JA and Horn JR. Comparative Pharmacology of Proton Pump Inhibitors. Managed Care, 2001; 10 (Suppl.): 11-16. 